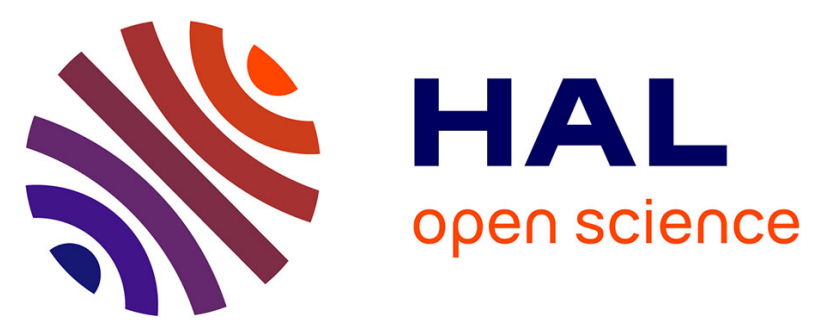

\title{
Modélisation bidimensionnelle dynamique du transistor à effet de champ MESFET : application à la conception de profils optimisés pour fonctionnement en faible bruit
}

F. Heliodore, G. Salmer, Y. Druelle, M. . Lefebvre, O. El Sayed

\section{- To cite this version:}

F. Heliodore, G. Salmer, Y. Druelle, M. . Lefebvre, O. El Sayed. Modélisation bidimensionnelle dynamique du transistor à effet de champ MESFET : application à la conception de profils optimisés pour fonctionnement en faible bruit. Revue de Physique Appliquée, 1988, 23 (7), pp.1185-1198. 10.1051/rphysap:019880023070118500 . jpa-00245930

HAL Id: jpa-00245930

https://hal.science/jpa-00245930

Submitted on 1 Jan 1988

HAL is a multi-disciplinary open access archive for the deposit and dissemination of scientific research documents, whether they are published or not. The documents may come from teaching and research institutions in France or abroad, or from public or private research centers.
L'archive ouverte pluridisciplinaire HAL, est destinée au dépôt et à la diffusion de documents scientifiques de niveau recherche, publiés ou non, émanant des établissements d'enseignement et de recherche français ou étrangers, des laboratoires publics ou privés. 


\title{
Modélisation bidimensionnelle dynamique du transistor à effet de champ MESFET : application à la conception de profils optimisés pour fonctionnement en faible bruit
}

\author{
F. Heliodore, G. Salmer, Y. Druelle, M. Lefebvre et O. El Sayed ( ${ }^{1}$ ) \\ Université des Sciences et Techniques de Lille Flandres Artois UA 287 CNRS Bâtiment P4 59655 Villeneuve \\ D'Ascq Cedex, France \\ (1) Department of Electronics, Cairo University, Cairo, Egypt
}

(Reçu le 2 novembre 1987, révisé le 18 mars 1988, accepté le 11 avril 1988)

\begin{abstract}
Résumé. - Un modèle de simulation numérique de transistor à effet de champ basé sur une résolution bidimensionnelle des équations des semiconducteurs est présenté. Il inclut les effets de dynamique non stationnaire et permet l'étude du comportement des composants tant en régime statique que dynamique (petit et grand signal, impulsionnel). Il est montré comment on peut accéder aux paramètres caractéristiques du régime petit signal, grâce à l'utilisation d'une transformation de Fourier rapide. Ce modèle est utilisé pour étudier l'influence du profil de dopage sur les éléments caractéristiques du comportement des composants et sur leurs performances potentielles. Il permet de mettre en évidence l'intérêt des composants à canal enterré tant pour l'amplification de puissance que pour l'amplification faible bruit, ce qui est clairement confirmé par les premiers résultats de l'étude expérimentale.
\end{abstract}

\begin{abstract}
A GaAs MESFET model, based on the two dimensional resolution of the semiconductor hydrodynamic equations, is presented. It includes a carrier energy relaxation equation, allowing to account for non stationary electron dynamic effects. It is shown that it is possible to obtain an accurate determination of the main equivalent circuit elements by using transient response and fast Fourier transform. This model is systematically used to study the influence of doping profile on device characteristics and the expected performances. The potential interest of burried channel devices is clearly shown under large signal and low noise conditions respectively. These conclusions are confirmed by experiment.
\end{abstract}

\section{Introduction.}

La prévision de performances potentielles fiables pour les dispositifs analogiques ou logiques utilisant des transistors à effet de champ nécessite de disposer de modèles aussi exacts que possible de ces composants. C'est particulièrement vrai pour les nouvelles générations de composants à grille submicronique devant être optimisés pour un fonctionnement en ondes millimétriques. Dans ceux-ci de nouveaux phénomènes découlant de la dynamique non stationnaire des porteurs de charge en modifient profondément le comportement $[1,2]$. En particulier, les effets de survitesse peuvent contribuer à une amélioration importante des performances potentielles quand les longueurs de grille deviennent très inférieures au micron. A l'opposé, le déplacement des porteurs a un caractère bidimensionnel de plus en plus marqué dans ce type de structure et les effets d'injection dans le substrat prennent une importance croissante.

Pour ces raisons, les modèles tant analytiques [3] que numériques [4-6], qui ne prennent pas en compte ces phénomènes ne peuvent permettre d'obtenir des prévisions indiscutables quant aux caractéristiques de ces transistors. Seuls les modèles basés sur le traitement microscopique de semiconducteurs tels que les résolutions itératives de l'équation de Boltzmann ou les méthodes Monte Carlo [711] qui tiennent compte des effets de dynamique non stationnaire des porteurs de charge, peuvent apporter des informations fiables quant au comportement de ces composants. Seulement, si l'on veut avoir une précision suffisante en particulier pour obtenir les éléments essentiels du schéma équivalent, il faut considérer un grand nombre de particules et les temps de calcul deviennent longs. De même, il est 
difficile d'envisager leur utilisation pour étudier le comportement du composant en régime dynamique impulsionnel ou sinusoïdal.

Nous avons proposé il y a quelques années un modèle qui tient compte des phénomènes de dynamique non stationnaire et qui est suffisamment simple pour être utilisable avec un microordinateur [12]. Il est basé sur une résolution unidimensionnelle des équations de relaxation de l'énergie moyenne et du moment de l'ensemble des porteurs. Bien qu'il fournisse une bonne prévision des performances pour des transistors à effet de champ planars de longueur de grille typiquement supérieure à $0,3 \mu$, la non prise en compte directe des effets bidimensionnels et d'autres effets parasites limite ses possibilités d'application pour des structures complexes.

Il nous a semblé intéressant de concevoir un nouveau modèle basé sur la résolution bidimensionnelle des équations fondamentales de transport dans un semiconducteur incluant les effets de relaxation de l'énergie moyenne des porteurs [12-16]. Une démarche un peu similaire a été proposée par Curtice [17], Frey et Cook [18], Brewitt-Taylor [19], Snowden et Loret [20]. Toutefois, les équations utilisées dans leurs simulations laissent apparaître un certain nombre d'approximations dont la justification n'est pas toujours évidente.

Signalons enfin qu'un formalisme basé sur l'utilisation des pseudo potentiels de Fermi est utilisé par de nombreux auteurs pour la modélisation de composants où l'on peut considérer que les effets de porteurs chauds peuvent être négligés. S. Mottet et al. ont montré qu'un tel formalisme était extensible au cas de porteurs chauds, moyennant certaines précautions liées à la forme des équations [21]. Ceci ouvre des perspectives nouvelles et constitue une alternative au modèle que nous proposons [22].

Dans la deuxième partie de cet article, nous résumerons les aspects du modèle décrit largement par ailleurs. Nous montrerons ensuite comment à partir de la réponse temporelle du composant on peut déduire avec une excellente précision les éléments du schéma équivalent en utilisant la transformée rapide de Fourier. Dans la quatrième partie, nous utiliserons ce modèle pour étudier l'influence du profil de dopage sur les performances potentielles de transistors à effet de champ à grille submicronique. Cette étude a suggéré l'emploi de profils dits $a$ canal enterré dont une première réalisation expérimentale sera décrite dans la dernière partie.

\section{Description du modèle.}

Ce modèle ayant été décrit largement par ailleurs et notamment dans [16], nous n'en rappellerons ici que les éléments essentiels et les plus significatifs.

2.1 ÉQUATIONS UTILISÉES. - Le modèle utilisé repose sur la résolution bidimensionnelle de trois équations fondamentales des semiconducteurs en utilisant une technique de discrétisation aux différences finies.

- Equation de Poisson

$$
\nabla^{2} V=-q \frac{N_{\mathrm{D}}-n}{\varepsilon_{0} \varepsilon_{\mathrm{r}}}
$$

- Equation de continuité

$$
\frac{\partial n}{\partial t}-\frac{1}{q} \nabla \cdot \mathbf{J}=0
$$

- Equation de conservation de l'énergie totale :

$$
\begin{aligned}
\frac{\partial(n w)}{\partial t}-\frac{1}{q} & \nabla(\mathbf{J} w)= \\
& =(\mathbf{J} \cdot \mathbf{E})+\nabla\left(\mathbf{J} \frac{k T(w)}{q}\right)-n \frac{w-w_{0}}{\tau_{w}(w)} .
\end{aligned}
$$

Le courant $J$ est défini par :

$$
\mathbf{J}=q \mu(w)\left[n \mathbf{E}+\frac{\nabla n k T(w)}{q}\right]
$$

où $w$ est l'énergie totale moyenne des porteurs et $\tau_{w}$ est le temps de relaxation de l'énergie, les autres termes ayant leur signification habituelle. Les équations de conservation de l'énergie et de définition du courant total sont obtenues par multiplication de l'équation de Boltzmann [23-25] par un terme approprié (moment $p_{\mathrm{i}}$, énergie cinétique $w_{\mathrm{i}}$ ), intégration sur chacune des vallées et sommation sur les trois vallées.

L'hypothèse fondamentale réside dans le fait que l'on postule que la distribution électronique ne dépend que de l'énergie totale moyenne de l'ensemble des électrons. En d'autres termes, que ce soit en régime stationnaire ou non stationnaire, l'énergie moyenne caractérise entièrement la distribution électronique et tous les paramètres qui en dépendent. Des comparaisons directes effectuées avec des résultats de simulation déduits du modèle Monte Carlo ont montré la validité de cette hypothèse [25]. Parmi les autres hypothèses nous pouvons citer :

- l'expression des termes de collision sous forme de temps de relaxation dépendant de l'énergie ;

- la suppresssion des effets inertiels en écrivant que :

$$
\frac{\partial p_{x i}}{\partial t}+\nabla\left(p_{x i}\left\langle\mathbf{v}_{\mathrm{i}}\right\rangle\right) \simeq 0
$$

Cette dernière approximation est utilisée par la plupart des auteurs [17-20]. Le premier terme peut entraîner quelques erreurs dans l'estimation des grandeurs locales $(n, V, W)$ en régime impulsionnel. Le second n'a une influence significative que sur les grandeurs locales $(n, W, V)$ pour des composants de longueurs de grille supérieures à $0,1 \mu$ [23] ; il devrait être pris en compte pour des longueurs de grille plus faibles [23]. 
Il faut noter que les fonctions $\mu(w), k t(w)$ et $\tau_{w}(w)$ sont déduites de calculs Monte Carlo effectués en régime statique pour un matériau homogène soumis à un champ électrique uniforme.

Prise en compte de l'influence du substrat. - Un grand nombre d'études tant théoriques qu'expérimentales ont montré $[26,27]$ l'étroite dépendance entre les propriétés électriques du substrat et les caractéristiques du composant.

Dans le cas de semi-isolants compensés au chrome ou non intentionnellement dopés, nous avons utilisé les conclusions des travaux effectués par S. Mottet et J. E. Viallet [28, 29] au CNET et par Makram-Ebeid au LEP [30] et avons introduit les termes correspondants dans le modèle afin d'étudier systématiquement leur influence [15].

Dans le cadre de cette étude, nous avons considéré le cas d'une couche tampon idéale en estimant que la dynamique électronique pouvait y être la même que dans la couche active, à ceci près que la mobilité bas champ y était plus importante $\left(\mu_{0}=5000 \mathrm{~cm}^{2} / \mathrm{V} \mathrm{s}\right.$ par exemple).

2.2 Conditions AUX Limites. - Nous utilisons tant pour les électrodes de source et de drain que pour les surfaces limites du semiconducteur les mêmes conditions que la plupart des auteurs précités. Sur les électrodes de source et de drain, ce sont les conditions de Dirichlet couramment admises et qui ne posent pas de problème physique particulier : $V=$ Cste, $n=N_{\mathrm{D}}, W=W_{0}$. Sur les autres surfaces limites du semiconducteur ce sont les conditions de Neumann.

Sur la grille, nous imposons que les deux composantes du courant (conduction et diffusion) qui rentrent sur la grille sont nulles. La concentration de porteurs en dessous de la grille évolue alors librement. Cette formulation donne des résultats beaucoup plus cohérents que l'utilisation d'autres conditions aux limites, mais elle limite actuellement l'utilisation du modèle aux régimes de polarisation de grille en inverse ou faiblement en direct.

En ce qui concerne le fond du substrat, nous avons admis que la couche tampon était suffisamment épaisse pour que les phénomènes se produisant à l'interface couche tampon semi-isolant ne soient pas perceptibles à l'interface avec la couche active. Dans ces conditions, il est inutile de considérer l'ensemble de la couche tampon. On prend en compte uniquement la zone où la concentration des porteurs n'est pas négligeable (typiquement $0,5 \mu$ pour une longueur de grille de $0,3 \mu$ ) et on utilise pour la limite de cette zone les conditions de Neumann.

2.3 Algorithme et MÉTHOdes de RÉSOlution NUMÉRIQUE UTILISÉs. - Nous réalisons ici une résolution temporelle bidimensionnelle des équations précitées.
Les différentes équations sont résolues à chaque intervalle de temps $\Delta t$ suivant l'algorithme représenté figure 1.

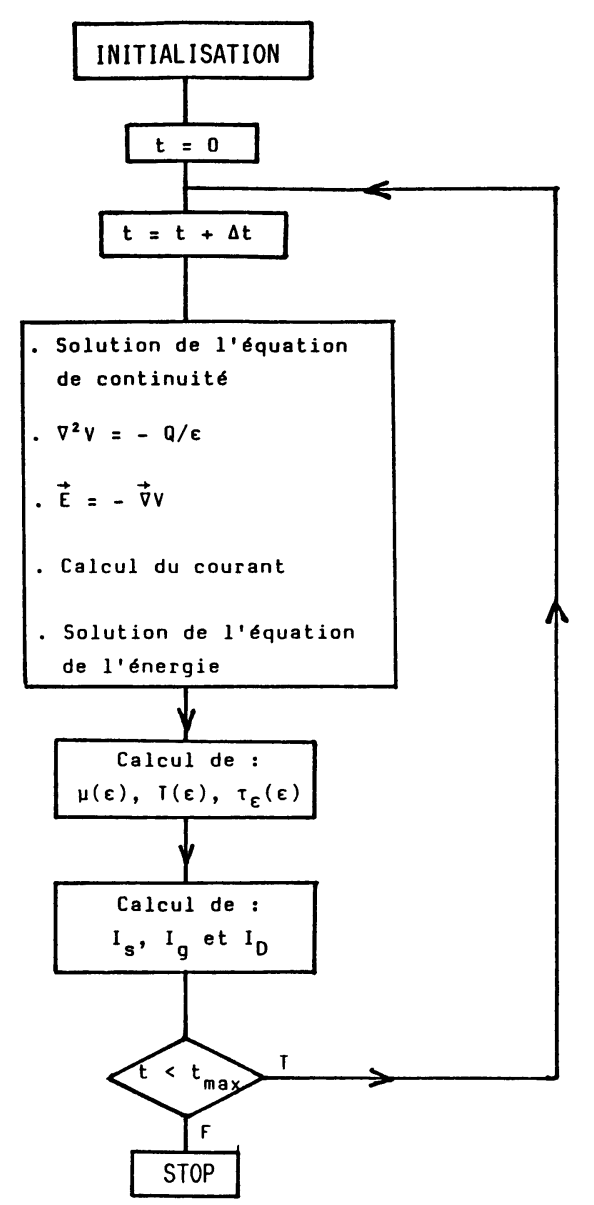

Fig. 1. - Organigramme de la simulation bidimensionnelle du transistor MESFET.

[Flow chart for simulation program.]

L'équation de Poisson est résolue par une méthode originale dite du double balayage matriciel qui est la généralisation de la méthode du double balayage ou de Choleski appliquée couramment à la résolution des équations différentielles du second ordre [24].

Les équations de continuité et de conservation de l'énergie sont écrites en formulation semi-implicite. Compte tenu de la différence importante entre le temps de relaxation diélectrique (ordre de $10^{-14} \mathrm{~s}$ dans la couche active) et celui de l'énergie (ordre de $10^{-12} \mathrm{~s}$ ), les résolutions de ces deux équations peuvent être découplées. Elles sont donc effectuées séparément par une méthode itérative de surrelaxation; en général cinq itérations sont nécessaires pour garantir une précision suffisante.

Les équations différentielles sont écrites sous forme de différences finies. Dans le cadre de cette étude, nous utilisons un maillage rectangulaire uniforme. Afin de rendre compte des particularités du 
composant à étudier (par exemple celui représenté Fig. 2), la largeur $\Delta y$ des mailles est souvent inférieure à la longueur $\Delta x$ : typiquement $\Delta y=80 \AA$ et $\Delta x=220 \AA$.

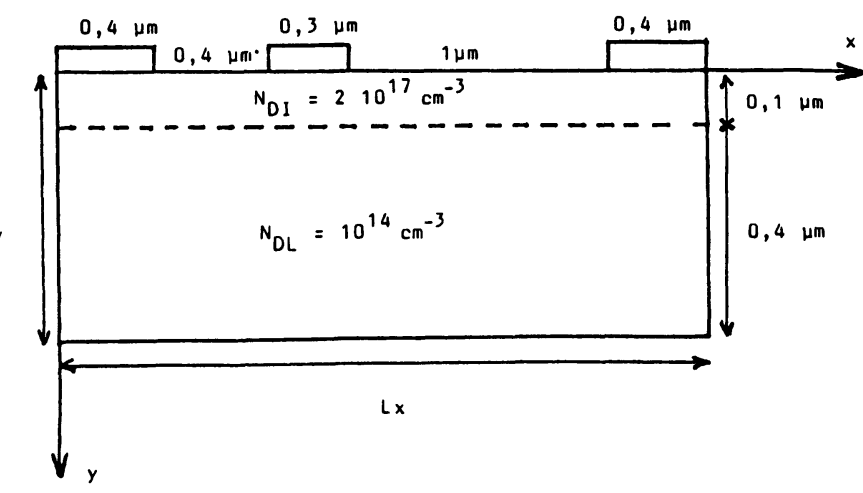

Fig. 2. - Composant typique simulé.

[Typical device simulated.]

2.4 ASPECTS PRATIQUES. - Ce modèle peut être utilisé pour prévoir les caractéristiques du composant en régime statique, ou la réponse du composant en régime impulsionnel ou dynamique petit et grand signal. Pour des valeurs données de tension drain source et grille drain, à partir des évolutions spatiales des principales grandeurs considérées (concentration de porteurs, potentiel, énergie, vitesse des porteurs) telles que celles représentées figure 3 , le programme calcule directement le courant drain. Les incréments de temps $\Delta t$ doivent dans tous les cas être inférieurs ou de l'ordre du temps de relaxation diélectrique si l'on veut une bonne stabilité de la résolution numérique : typiquement $10^{-14} \mathrm{~s}$ pour un dosage de $2 \times 10^{17}$ at. $/ \mathrm{cm}^{3}$. Compte tenu de la valeur du temps de relaxation de l'énergie, l'obtention du régime statique nécessite la prise en compte de la réponse à un échelon de tension durant 3 à 6 ps, ce qui correspond à 300 à 600 incréments de temps. Le programme de simulation numérique est actuellement implanté sur Bull DPS8 au CITI de Lille et sur IBM 3090 au CIRCE. Sur ce dernier matériel, l'obtention d'un point de la caractéristique statique nécessite environ $3 \mathrm{~min}$ de temps CPU (soit 6 ps en réponse impulsionnelle).

Signalons que la méthode de simulation supporte sans problème d'instabilités des échelons de tension de l'ordre de $2 \mathrm{~V}$ pour $V_{\text {ds }}$ et de $0,5 \mathrm{~V}$ pour $V_{\text {gs }}$.

Ce modèle a été utilisé par ailleurs pour étudier l'influence de la longueur de grille [13, 14], des propriétés du substrat $[15,16]$ et du potentiel de surface $[31,32]$ sur les caractéristiques et les performances potentielles de MESFET à grille submicronique. Son domaine d'application a été récemment étendu au cas du MODFET [33]. Dans le cadre de ce
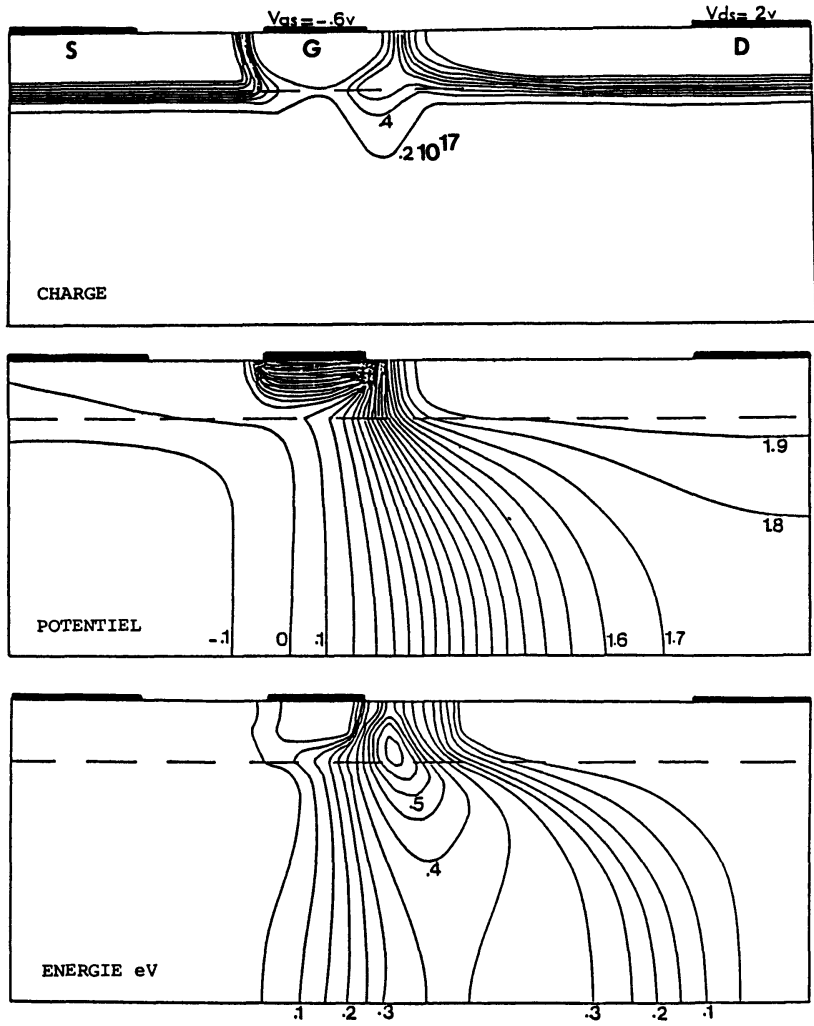

Fig. 3. - Evolutions spatiales de la concentration des porteurs, du potentiel et de l'énergie moyenne.

[Equicharge density, equipotential, and equienergy contours.]

travail, il a été utilisé pour étudier l'influence du profil de dopage sur les caractéristiques et les performances potentielles de MESFETs à grille submicronique utilisés surtout en régime petit signal faible bruit.

\section{Extraction des paramètres petit signal : utilisation de la transformée de Fourier rapide.}

Le comportement du transistor MESFET en régime petit signal est le plus souvent caractérisé par les valeurs des éléments du schéma équivalent dont le plus typique est représenté figure 4. Les éléments essentiels de ce schéma (transconductance $g_{\mathrm{m}}$,

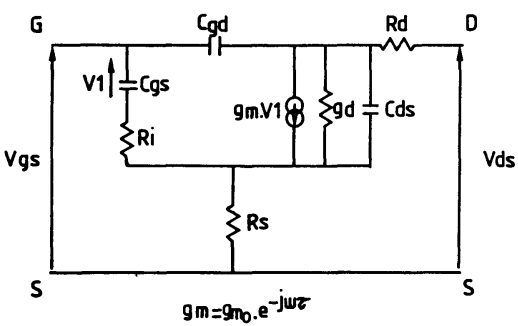

Fig. 4. - Schéma équivalent petit signal du transistor MESFET.

[Small signal equivalent scheme of MESFET.] 
conductance de drain $g_{\mathrm{d}}$, capacité d'entrée $C_{\mathrm{gs}}$, capacité de réaction $C_{\mathrm{gd}}$ ) peuvent être déduits à partir de l'étude réalisée en régime statique en imposant de petites variations $\Delta V_{\text {ds }}$ et $\Delta V_{\text {gs }}$ aux tensions drain source et grille source, et en relevant les variations correspondantes des courants et des charges sur les électrodes :

$$
\begin{aligned}
g_{\mathrm{m}} & =\frac{\Delta I_{\mathrm{d}}}{\Delta V_{\mathrm{gs}}} \text { à } V_{\mathrm{ds}} \text { constante } \\
g_{\mathrm{d}} & =\frac{\Delta I_{\mathrm{d}}}{\Delta V_{\mathrm{ds}}} \text { à } V_{\mathrm{gs}} \text { constante } \\
C_{\mathrm{gs}} & =\frac{\Delta Q_{\mathrm{g}}}{\Delta V_{\mathrm{gs}}} \text { à } V_{\mathrm{ds}} \text { constante } \\
C_{\mathrm{gd}} & =\frac{\Delta Q_{\mathrm{g}}}{\Delta V_{\mathrm{gd}}} \text { à } V_{\mathrm{gs}} \text { constante }
\end{aligned}
$$

$Q_{\mathrm{g}}$ est la charge apparaissant sur la grille.

Toutefois, il n'est pas certain que de tels paramètres représentent bien le comportement haute fréquence du transistor. De plus cette méthode ne permet pas d'évaluer certains éléments du schéma équivalent tels que la résistance d'accès de grille $R_{\mathrm{i}}$, la capacité $C_{\mathrm{ds}}$ et la constante de temps $\tau$ de la transconductance $g_{\mathrm{m}}\left(g_{\mathrm{m}}=g_{\mathrm{mo}} \mathrm{e}^{-j \omega \tau}\right)$. Rappelons que cette constante de temps $\tau$ dépend des temps de transit $\tau_{t}$ des porteurs sous la grille. Des formulations approchées donnent $\tau \not \# \tau_{t} / 3$.

Il nous a semblé préférable de calculer les paramètres essentiels du schéma équivalent à partir de la dépendance fréquentielle des paramètres admittance $\mathrm{du}$ quadripole équivalent aux composants, obtenus eux-mêmes par transformation de Fourier rapide à partir de sa réponse transitoire.

3.1 PRINCIPES DE LA DÉTERMINATION. - Les paramètres $y_{i j}$ peuvent être obtenus à partir de la formulation suivante [34]

$$
y_{i j}=\frac{F\left(i_{i}(t)-i_{i}(\mathrm{o})\right)}{F\left(v_{j}(t)-v_{j}(\mathrm{o})\right)}
$$

où les indices $i$ et $j$ représentent le quadripôle, $i_{i}(t)$ est la réponse temporelle sur l'électrode $i$ à l'application d'une tension $v_{j}(t)$ sur l'électrode $j$ et $F$ est le symbole de la transformée de Fourier.

Si l'on applique un échelon unité d'amplitude $\Delta V_{j}$, on obtient [26] :

$$
y_{i j}=\frac{I_{i}(\alpha)-I_{i}(\mathrm{o})}{\Delta V_{j}}+j \omega \frac{F\left[i_{i}(t)-I_{i}(\alpha)\right]}{\Delta V_{j}}
$$

où $I_{i}(\mathrm{o})$ et $I_{i}(\alpha)$ représentent respectivement les valeurs initiales et finales du courant.

Les parties réelles et imaginaires des quatre paramètres admittance peuvent être calculées à partir de ces formules.

Par ailleurs on peut trouver dans certaines gammes de fréquences des relations directes entre les paramè- tres $y_{i j}$ et les éléments du schéma équivalent du transistor :

$$
\begin{aligned}
& y_{11} \# \omega^{2} C_{\mathrm{gs}^{2}}\left(R_{\mathrm{i}}+R_{\mathrm{s}}\right)+j \omega\left(C_{\mathrm{gs}}+C_{\mathrm{gd}}\right) \\
& y_{12} \#-j \omega C_{\mathrm{gd}} \\
& y_{21} \# g_{\mathrm{mo}}-j \omega\left(C_{\mathrm{gd}}+g_{\mathrm{mo}}\left(\tau+\left(R_{\mathrm{s}}+R_{\mathrm{i}}\right) C_{\mathrm{gs}}\right)\right) \\
& y_{22} \# g_{\mathrm{d}}+j \omega\left(C_{\mathrm{gd}}+C_{\mathrm{ds}}\right)
\end{aligned}
$$

à condition que $\left(g_{\mathrm{d}}+g_{\mathrm{m}}\right) R_{\mathrm{s}} \ll 1$ et que $\left(R_{\mathrm{i}} C_{\mathrm{gs}} \omega\right)^{2} \ll 1$.

La première condition est bien vérifiée en pratique quand on simule la zone active du transistor ( $R_{\mathrm{s}}$ est très faible): la deuxième condition est satisfaite en basse fréquence.

On peut remarquer que les dépendances fréquentielles des parties réelles et imaginaires des paramètres $y_{i j}$ sont suffisamment simples (constante, linéaire, quadratique) pour permettre par la méthode des moindres carrés un calcul direct des sept coefficients inconnus dont on extraira les valeurs des sept éléments du schéma équivalent.

L'utilisation de ces résultats est triple :

- on peut vérifier la validité des formulations approchées à partir de la forme de l'évolution fréquentielle des paramètres $y_{i j}$;

- on peut comparer les valeurs des éléments du schéma équivalent obtenus à ceux déduits de l'étude quasi statique et voir dans quelle mesure ceux-ci sont bien représentatifs du comportement fréquentiel du transistor ;

- on peut évaluer d'autres paramètres tels que $R_{\mathrm{i}}, \tau, C_{\mathrm{ds}}$, etc.

3.2 RÉSUltATS TYPIQUES OBTENUS. - Nous avons obtenu l'ensemble des paramètres $y_{i j}$ pour le composant décrit précédemment (Fig. 2). On considère que le courant grille est uniquement un courant de déplacement. Nous présentons à titre d'exemple figure 5 les évolutions des parties réelles et imaginaires des paramètres $y$ de sortie $\left(y_{21}\right.$ et $\left.y_{22}\right)$. On. peut vérifier que dans la gamme de fréquences et pour le point de fonctionnement considéré $\left(V_{\mathrm{ds}}=2 \mathrm{~V}\right.$ et $\left.V_{\mathrm{gs}}=-0,15 \mathrm{~V}\right)$, les approximations précédentes (en particulier $\left(R_{\mathrm{i}} C_{\mathrm{gs}} \omega\right)^{2} \ll 1$ ) sont bien vérifiées.

Le tableau I donne un exemple des valeurs des éléments du schéma équivalent obtenus en utilisant cette méthode. Par comparaison, figurent également celles déduites d'une étude quasi statique. On peut remarquer que les valeurs obtenues par les deux méthodes sont très voisines, ce qui confirme leur validité. De plus cette méthode nous fournit des informations très précises sur les valeurs de $R_{\mathrm{i}}$, $C_{\mathrm{ds}}$ et $\tau$.

Si l'on soustrait de la valeur de $R_{\mathrm{s}}+R_{\mathrm{i}}$ obtenue celle de la résistance présentée par le matériau entre source et grille, soit $0,23 \Omega / \mathrm{mm}$, on obtient une valeur de $R_{\mathrm{i}}$ de l'ordre de $0,25 \Omega / \mathrm{mm}$ : elle est proche de $R_{0} / 3$ ( $R_{0}$ résistance du canal ouvert), valeur couramment admise loin du pincement. 
Tableau I. - Comparaison des valeurs des paramètres petit signal obtenus à partir de la caractéristique statique et de la transformée de Fourier respectivement.

[Calculation of the MESFET equivalent scheme elements : comparison between values deduced from static behaviour and from transient response (by using fast Fourier transform).]

\begin{tabular}{|l|c|c|}
\hline \multicolumn{2}{|c|}{ Point de fonctionnement $V_{\mathrm{gs}}=-0,15 \mathrm{~V}$ et $V_{\mathrm{ds}}=2 \mathrm{~V}$} \\
\hline & Quasi statique & $\begin{array}{c}\text { Eléments déduits } \\
\text { par transformée } \\
\text { de Fourier }\end{array}$ \\
& & 216 \\
$g_{\mathrm{m}}(\mathrm{mS} / \mathrm{mm})$ & 220 & 15,5 \\
$g_{\mathrm{d}}(\mathrm{mS} / \mathrm{mm})$ & 16 & 0,46 \\
$C_{\mathrm{gs}}(\mathrm{pF} / \mathrm{mm})$ & 0,48 & 20 \\
$C_{\mathrm{gd}}(\mathrm{FF} / \mathrm{mm})$ & 17 & $1,35 \mathrm{pS}$ \\
$\tau$ & & $0,48 \Omega / \mathrm{mm}$ \\
$R_{\mathrm{o}}+R_{\mathrm{i}}$ & & $13 \quad \mathrm{FF} / \mathrm{mm}$ \\
$C_{\mathrm{ds}}$ & & \\
\hline
\end{tabular}

La valeur de la capacité drain source dont la cause couramment admise réside dans l'existence du domaine d'électrons chauds à la sortie de la grille est ici de $13 \mathrm{FF} / \mathrm{mm}$. Elle est très inférieure à la plupart des déterminations expérimentales. Ceci montre clairement qu'en pratique $C_{\mathrm{ds}}$ est principalement d'origine électrostatique et qu'elle est surtout due aux métallisations et interconnections.

La constante de temps $\tau$, de l'ordre de 1,35 ps est proche des déterminations expérimentales pour des composants de ces dimensions. Elle conduit à une fréquence de transit supérieure à $100 \mathrm{GHz}$, soit notablement supérieure à la fréquence de coupure du gain en courant.

Cet exemple typique nous a permis de montrer clairement les possibilités de cette méthode d'évaluation des éléments du schéma équivalent, et la validité de la détermination quasi statique. Ce
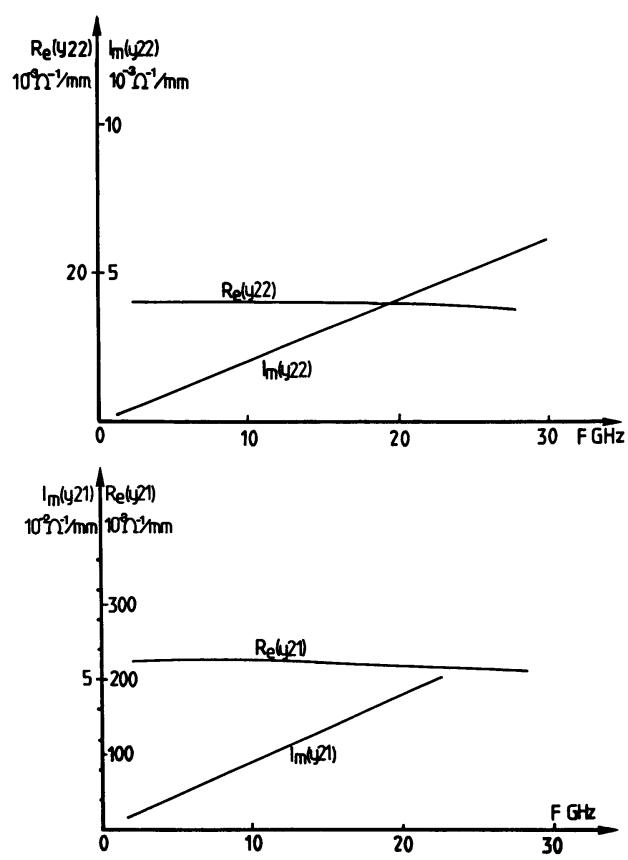

Fig. 5. - Evolution des parties réelles et imaginaires de $y_{21}(5 \mathrm{a})$ et $y_{22}(5 \mathrm{~b})$.

[Frequency variations of $y_{21}(5 \mathrm{a})$ and $y_{22}(5 \mathrm{~b})$ parameters.]

modèle a pu ainsi être utilisé pour étudier l'influence $\mathrm{du}$ profil de dopage sur les performances.

\section{Etude théorique des profils de dopage.}

L'influence du profil de dopage sur le comportement et les performances potentielles d'un transistor peuvent être précisées à partir des évolutions des paramètres caractéristiques du composant en particulier les éléments du schéma équivalent $\left(g_{\mathrm{m}}\right.$, $\left.g_{\mathrm{d}}, C_{\mathrm{gs}}, C_{\mathrm{gd}}\right)$ et la fréquence de coupure $f_{\mathrm{c}}=$ $g_{\mathrm{m}} / 2 \pi C_{\mathrm{gs}}$. Il nous a paru intéressant d'utiliser des formulations approchées du gain maximum disponible [35] et du facteur de bruit [32-37]. Bien que la validité de cette dernière puisse être discutée, elle permet d'en obtenir de façon générale une bonne estimation :

$$
\begin{aligned}
& \left.G=\left(\frac{f_{\mathrm{c}}}{\mathrm{F}}\right)^{2} \frac{1}{4 g_{\mathrm{d}}\left(R_{\mathrm{o}}+\pi f_{\mathrm{c}} L_{\mathrm{s}}\right)+4 \pi f_{\mathrm{c}} C_{\mathrm{gd}}\left(R_{\mathrm{o}}+R_{\mathrm{g}}+2 \pi f_{\mathrm{c}} L_{\mathrm{s}}\right.}\right) \\
& N_{F}=1+\sqrt{8 \pi F}\left[L_{\mathrm{g}} / f_{\mathrm{c}}\left(\alpha Z+\beta I_{\mathrm{ds}}\right)\left(R_{\mathrm{s}}+R_{\mathrm{g}}\right)\left(1+\omega^{2} C_{\mathrm{gd}^{2} / g_{\mathrm{d}}^{2}}\right)\right]^{1 / 2}
\end{aligned}
$$

où

$$
R_{\mathrm{o}}=R_{\mathrm{i}}+R_{\mathrm{s}}+R_{\mathrm{g}}
$$

$L_{\mathrm{s}}$ self de connexion de source

$Z$ largeur du composant

$$
\alpha=2 \times 10^{-3} \mathrm{SI} \text { et } \beta=1,25 \times 10^{-5} \mathrm{SI} .
$$

A partir de ces expressions, nous voyons que les paramètres importants pour le gain et le facteur de bruit seront la fréquence de coupure et les rapports $f_{\mathrm{c}} / \sqrt{g_{\mathrm{d}}}$ et $C_{\mathrm{gd}} / g_{\mathrm{d}}$.
L'évolution de ces différentes grandeurs sera considérée en conditions forte puissance $\left(I_{\mathrm{d}} \# I_{\mathrm{dss}} / 2\right)$ et faible bruit $\left(I_{\mathrm{d}} \# 0,15 I_{\mathrm{dss}}\right)$.

4.1 DESCRIPTION DES STRUCTURES. - Trois types de profil correspondant aux possibilités technologiques actuelles ont été simulées (Fig. 6) :

- le profil de dopage uniforme déjà envisagé au paragraphe précédent: $N_{\mathrm{D}}=2 \times 10^{17}$ at. $/ \mathrm{cm}^{3}$, $a=0,1 \mu$; 

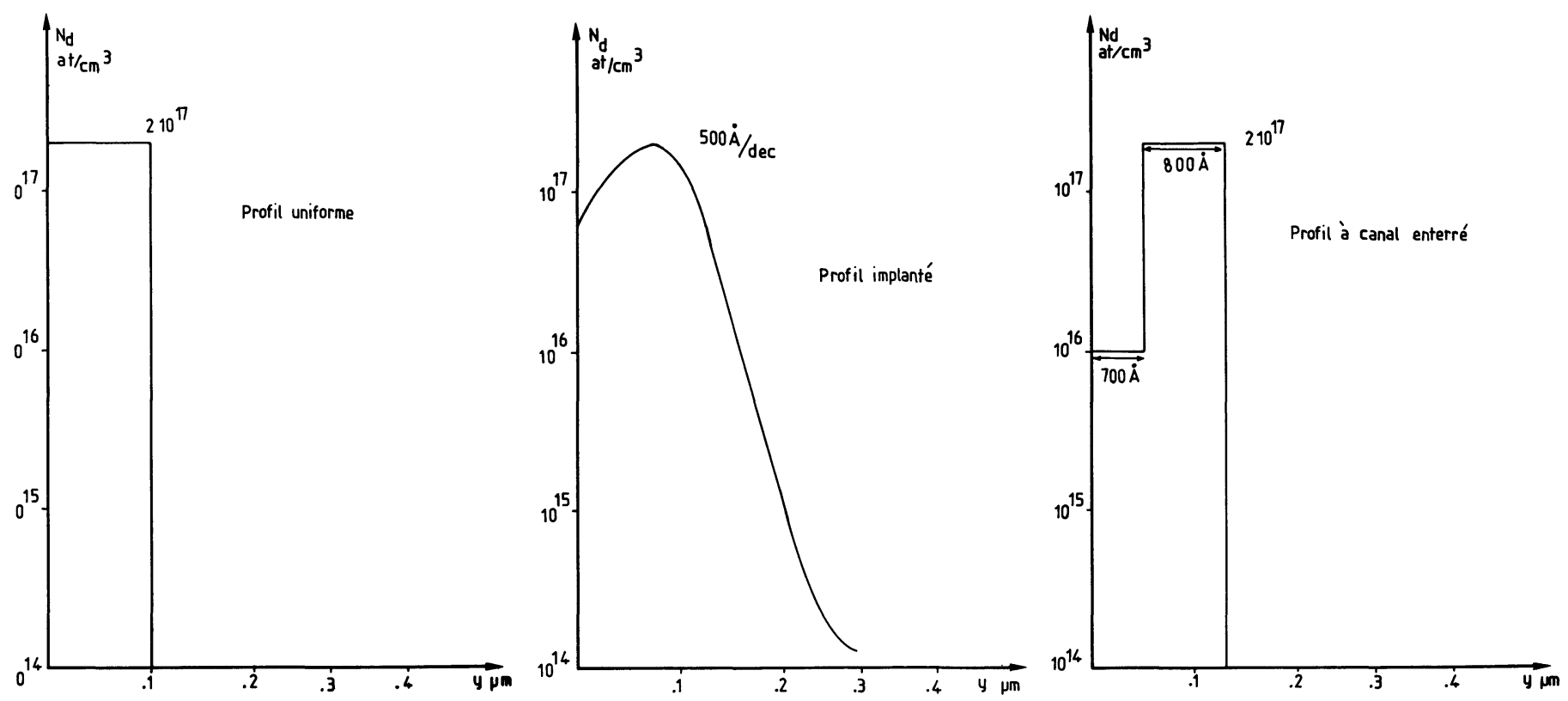

Fig. 6. - Profils de dopage simulés.

[Considered doping profiles of MESFET.]

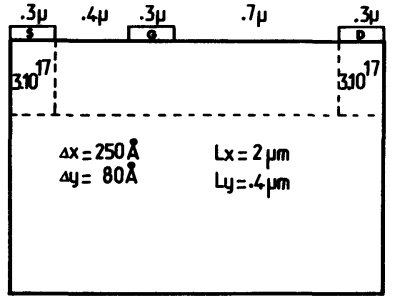

Fig. 7. - Composant simulé.

[Dimensions and technological characteristics of simulated MESFET structures.]

- le profil gaussien obtenu par implantation ionique et attaque chimique classique ;

- le profil à canal enterré [38] obtenu directement par épitaxie par exemple à jets moléculaires.

La charge totale équivalente est du même ordre de grandeur que dans le transistor à profil de dopage uniforme. Pour améliorer les contacts et réduire les résistances d'accès, les régions sous la source et le drain sont surdopées (Fig. 7).

4.2 RÉSUltATS TYPIQUES OBTENUS. - Sur les figures $8,9,10$ et 11 , nous avons représenté la caractéristique $I_{\mathrm{d}}=f\left(V_{\mathrm{gs}}\right)$ et les évolutions de la transconductance, de la capacité d'entrée et de la fréquence de coupure en fonction de la tension $V_{\mathrm{gs}}$.

Ces résultats montrent clairement que le transistor utilisant un profil à canal enterré présente une linéarité supérieure à celle des autres composants. Certes, pour un fonctionnement à canal ouvert, ou

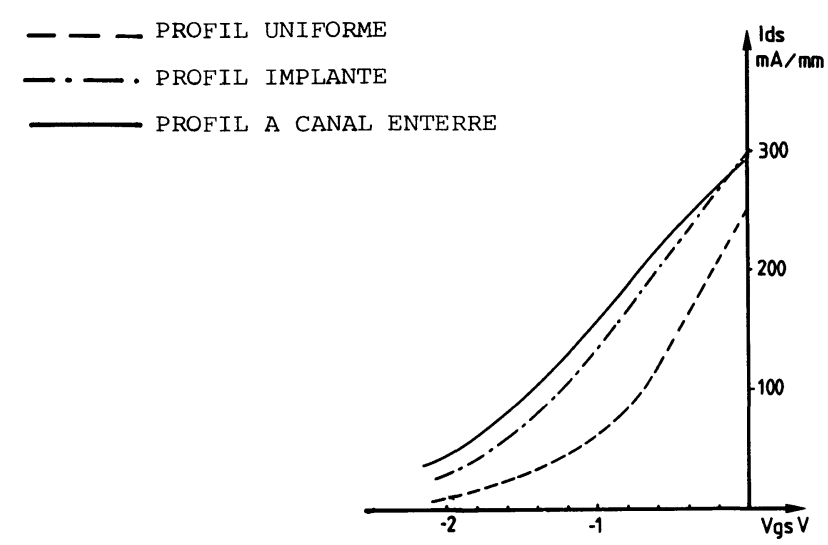

Fig. 8. - Evolution du courant $I_{\mathrm{ds}}$ en fonction de la tension $V_{\mathrm{gs}}$ pour les trois profils étudiés $\left(V_{\mathrm{ds}}=2 \mathrm{~V}\right)$.

[Theoretical variations of drain current $I_{\mathrm{ds}}$ as function of gate to source voltage $V_{\mathrm{gs}}$ for the three doping profiles considered $\left(V_{\mathrm{ds}}=2 \mathrm{~V}\right)$.]

en polarisation de courant élevé, le transistor à profil uniforme présente des caractéristiques beaucoup plus intéressantes, que ce soit en transconductance ou en fréquence de coupure. Mais dans tous les autres cas et en particulier dans les conditions faible bruit, le transistor à canal enterré semble de loin le plus intéressant.

La différence de comportement des différents types de composants peut s'expliquer aisément en considérant l'épaisseur de zone désertée équivalente $W_{\text {eq }}$ et ses variations ; elles sont caractéristiques du 


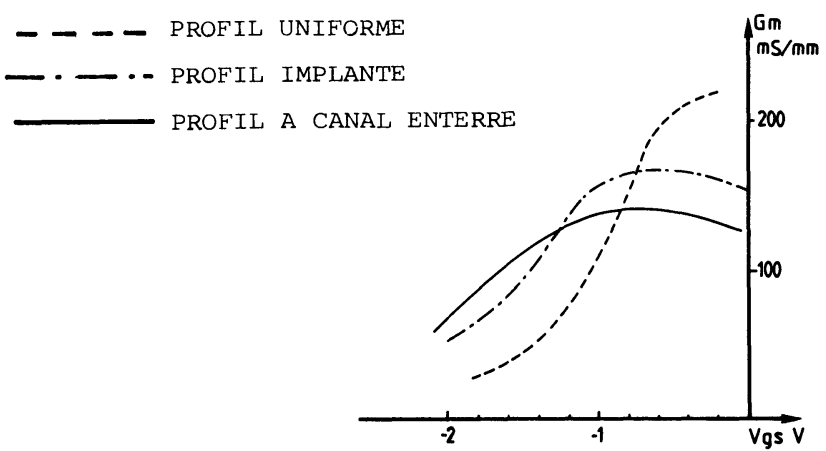

Fig. 9. - Evolution de la transconductance $g_{\mathrm{m}}$ en fonction de la tension $V_{\mathrm{gs}}$ pour les trois profils étudiés $\left(V_{\mathrm{ds}}=\right.$ $2 \mathrm{~V})$.

[Theoretical variations of transconductance $g_{\mathrm{m}}$ as function of $V_{\mathrm{gs}}$ in the same conditions as in figure 8.]

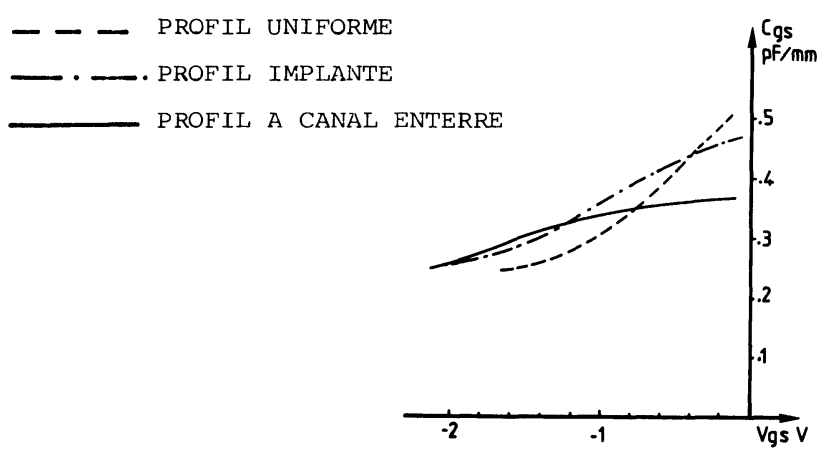

Fig. 10. - Evolution de la capacité d'entrée $C_{\mathrm{gs}}$ en fonction de la tension $V_{\mathrm{gs}}$ pour les trois profils étudiés $\left(V_{\mathrm{ds}}=2 \mathrm{~V}\right)$.

[Theoretical variations of gate to source capacitance $C_{\mathrm{gs}}$ as a function of $V_{\mathrm{gs}}$ in the same conditions as in figure 8.]

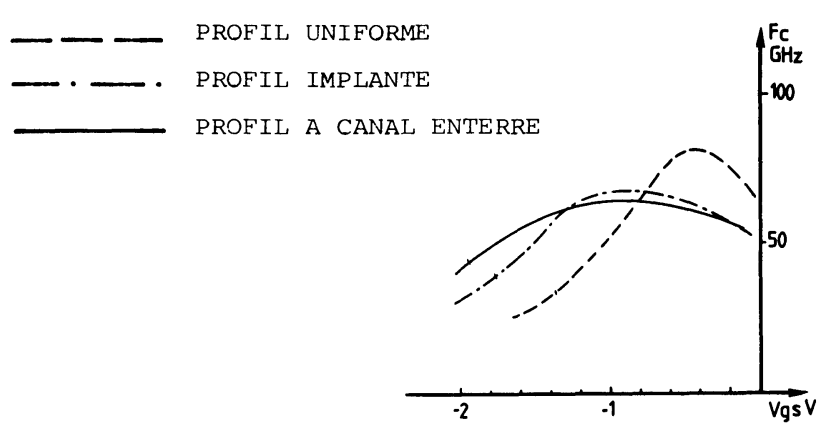

Fig. 11. - Evolution de la fréquence de coupure $f_{\mathrm{c}}$ en fonction de la tension $V_{\mathrm{gs}}$ pour les trois profils étudiés $\left(V_{\mathrm{ds}}=2 \mathrm{~V}\right)$.

[Cut off frequency $f_{\mathrm{c}}$ variations as function of $V_{\mathrm{gs}}$ in the same conditions as in figure 8.]

contrôlé exercé par la grille et figurent au dénominateur des formules approchées de $g_{\mathrm{m}}$ et $C_{\mathrm{gs}}$

$$
g_{\mathrm{m}} \# \frac{\varepsilon Z\langle v\rangle}{W_{\mathrm{eq}}} \text { et } C_{\mathrm{gs}} \# \frac{\varepsilon Z L_{\mathrm{g}}}{W_{\mathrm{eq}}} \text {. }
$$

La meilleure linéarité du profil enterré provient du fait que l'épaisseur $W_{\text {eq }}$ varie beaucoup moins quand $V_{\mathrm{gs}}$ varie.

4.3 INFLUENCE D'UN SURDOPAGE. - La structure à canal enterré est pénalisée par l'importance des résistances d'accès à la zone active. Afin de les réduire, il nous a semblé intéressant de surdoper la plus grande partie des zones grille source et source drain (Fig. 12). Conformément aux prévisions données par un raisonnement qualitatif, on note une amélioration importante de la transconductance (Fig. 13) sans pour autant, ce qui est très intéressant, relever une dégradation significative de la capacité d'entrée. Ceci montre bien l'intérêt potentiel de telles structures. Il faut noter que l'augmentation importante de la transconductance est liée non seulement à la diminution de la résistance d'accès mais aussi à l'augmentation de la vitesse moyenne des porteurs, ce qui est confirmé par l'analyse du diagramme des vitesses: les porteurs parviennent sous la grille avec une énergie moyenne plus petite (ils sont plus froids), leur mobilité est meilleure est leur vitesse moyenne plus élevée.

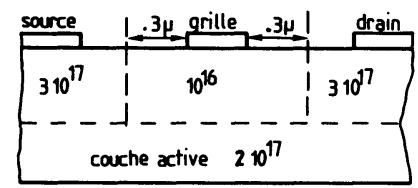

Fig. 12. - Composant simulé avec zones de surdopage source et drain.

[Dimensions and technological parameters of MESFET with overdopped source and drain zones.]

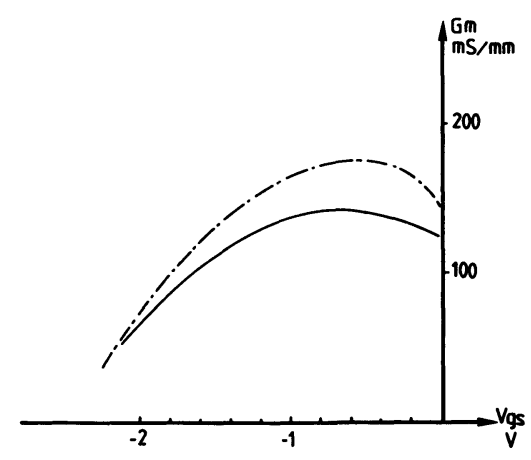

Fig. 13. - Evolution de la transconductance $g_{\mathrm{m}}$ en fonction de la tension $V_{\mathrm{gs}}$ pour la structure à canal enterré. - sans surdopage $;-$ - - avec surdopage.

[Theoretical transconductance variations as function of $V_{\mathrm{gs}}$ for the buried channel MESFET : influence of overdoping. - device represented figure $7 ;-\cdot-$ device represented figure 12.] 


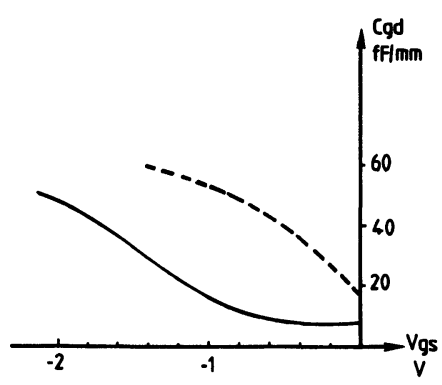

Fig. 14. - Evolution de la capacité de réaction $C_{\mathrm{gd}}$ en fonction de la tension $V_{\mathrm{gs}}$ pour la structure à profil uniforme - - ; pour la structure à canal enterré

[Comparison between feedback capacitance $C_{\mathrm{gd}}$ variations as function of $V_{\mathrm{gs}}:--\longrightarrow$ uniform doping profile ; buried channel.]
Il est évident que les conclusions essentielles des comparaisons faites avec le profil de dopage uniforme restent vraies, quand on introduit ce surdopage supplémentaire. Un point particulièrement important quand on envisage une application faible bruit en ondes millimétriques réside dans la valeur de la capacité de réaction $C_{\mathrm{gd}}$ qui intervient directement dans le facteur de bruit. Comme le montre la figure 14 celle-ci est beaucoup plus faible pour la structure à canal enterré, que ce soit à canal ouvert ou en conditions faible bruit. Ces différences de comportement peuvent être expliquées en considérant deux éléments :

- l'existence d'une zone moins dopée sous la grille ;

- le domaine d'électrons chauds est plus proche du drain pour les profils à canal enterré comme le montrent les courbes équi-énergie (Fig. 15).
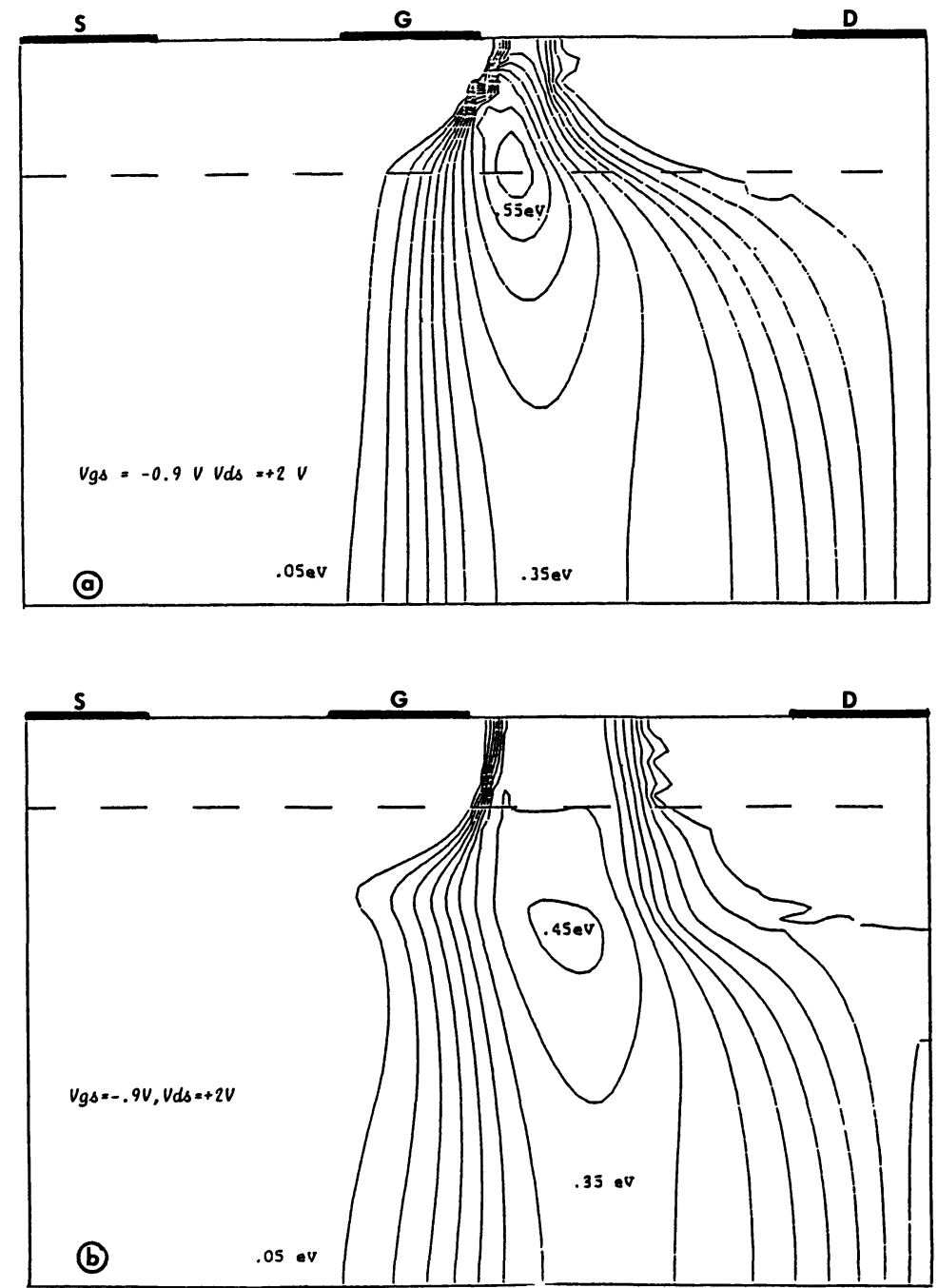

Fig. 15. - Répartition spatiale de l'énergie moyenne des porteurs : a) profil uniforme ; b) profil à canal enterré avec surdopage.

[Comparison between equienergy contours : a) uniform doping profile ; b) buried channel with overdoped zones close to source and drain contacts (Fig. 12).] 
4.4 CONCLUSION : INTÉRÊT DU CANAL ENTERRÉ. L'intérêt du profil à canal enterré apparaît nettement sur les tableaux II et III. Dans le premier sont résumées les caractéristiques essentielles des quatre types de composants tant en conditions de fonctionnement en puissance qu'en conditions faible bruit.

Dans le second, sont données des estimations du facteur de bruit pour les quatre profils aux fréquences de 20,40 et $60 \mathrm{GHz}$ pour des transistors à utiliser typiquement en ondes millimétriques $(Z=75 \mu \mathrm{m}$, $R_{\mathrm{s}}+R_{\mathrm{g}} \# 4,6 \Omega$ ). Même si les formulations précédentes ne sont qu'approchées et si en particulier la formule du facteur de bruit donne des estimations un peu optimistes, elles fournissent des indications précieuses à titre comparatif.

Quel que soit le critère que l'on utilise, que ce soit en fonctionnement en puissance ou en conditions faible bruit, la supériorité du profil à canal enterré avec surdopage dans les espaces grille source et grille drain apparaît nettement. Il faut noter par ailleurs que pour le fonctionnement en puissance, deux autres éléments contribuent à l'utilisation d'un tel profil : la linéarité du gain et la capacité d'obtention d'une tension de claquage plus élevée à cause de l'existence d'un faible dopage sous la grille.

$\mathrm{Vu}$ l'intérêt de telles structures, il nous a semblé important de voir si une approche expérimentale même partielle pouvait confirmer les conclusions de l'étude théorique.

\section{Etude expérimentale des composants à canal} enterré.

Pour cette étude expérimentale, deux séries de composants ont été conçues et réalisées par la Centrale de Technologie du Laboratoire.

5.1 DESCRIPTION DES COMPOSANTS. - Les couches ont été élaborées par épitaxie par jets moléculaires. A partir d'un substrat semi-isolant, on fait croître une couche tampon de type $\pi$ sur une épaisseur de $1 \mu$. Les caractéristiques de la couche active sont :

- pour la structure de référence : un dopage de $4 \times 10^{17} \mathrm{at} / \mathrm{cm}^{3}$ sur une épaisseur de $2000 \AA$;

- pour le composant à canal enterré : un dopage de $6 \times 10^{17} \mathrm{at} / \mathrm{cm}^{3}$ sur $800 \AA$ et de $1,5 \times 10^{16}$ at. $/ \mathrm{cm}^{3}$ sur une épaisseur de $600 \AA$.

Tableau II. - Principales caractéristiques et valeur des éléments du schéma équivalent pour les quatre types de composants étudiés: MP conditions de forte puissance: $I_{\mathrm{d}} \# I_{\mathrm{dss} / 2}$. FB conditions de faible bruit: $I_{\mathrm{d}} \# 0,15 I_{\mathrm{dss}}$.

[Typical values of equivalent scheme elements for the four kinds of devices considered.]

\begin{tabular}{|c|c|c|c|c|c|c|c|c|c|c|c|c|c|c|c|}
\hline $\begin{array}{l}\text { MP : } I_{\text {dss }} / 2 \\
\text { FB }: 0.15 I_{\text {dss }}\end{array}$ & $\begin{array}{c}I_{\mathrm{dss}} \\
\mathrm{mA} / \mathrm{mm}\end{array}$ & \multicolumn{2}{|c|}{$\begin{array}{c}\begin{array}{c}g_{\mathrm{m}} \\
\mathrm{mS} / \mathrm{mm}\end{array} \\
\mathrm{MP} \quad \mathrm{FB}\end{array}$} & \multicolumn{2}{|c|}{$\begin{array}{c}\begin{array}{c}C_{\mathrm{gs}} \\
\mathrm{pF} / \mathrm{mm}\end{array} \\
\mathrm{MP} \quad \mathrm{FB}\end{array}$} & \multicolumn{2}{|c|}{$\begin{array}{c}C_{\mathrm{gd}} \\
\mathrm{FF} / \mathrm{mm}\end{array}$} & \multicolumn{2}{|c|}{$\underset{\mathrm{mS} / \mathrm{mm}}{g_{\mathrm{d}}}$} & $\begin{array}{c}f_{\mathrm{c}} \\
\mathrm{GHz}\end{array}$ & FB & \multicolumn{2}{|c|}{$\begin{array}{c}f_{\mathrm{c}} / \sqrt{G_{\mathrm{d}}} \\
10^{10}(\mathrm{SI})\end{array}$} & \multicolumn{2}{|c|}{$\begin{array}{c}C_{\mathrm{gd}} / G_{\mathrm{d}} \\
10^{-3}(\mathrm{SI})\end{array}$} \\
\hline Profil uniforme & 250 & 200 & 55 & 0,41 & 0,265 & 40 & 56 & 19 & 12 & 78 & 33 & 56,6 & 30 & 21 & 46,7 \\
\hline Profil implanté & 296 & 162 & 64 & 0,375 & 0,265 & 25 & 46 & 19 & 11 & 68 & 39 & 49 & 37 & 13,2 & 42 \\
\hline Profil à canal enterré & 292 & 135 & 69 & 0,34 & 0,255 & 11 & 42 & 13 & 12,5 & 63 & 43 & 55,3 & 38,5 & 8,5 & 33,6 \\
\hline $\begin{array}{l}\text { Profil à canal enterré } \\
\text { avec surdopage des } \\
\text { régions d'accès }\end{array}$ & 335 & 170 & 76 & 0,35 & 0,26 & 12 & 47 & 14 & 14 & 77 & 47 & 65 & 39,8 & 8,6 & 33,6 \\
\hline
\end{tabular}

Tableau III. - Facteur de bruit estimé pour les quatre types de composants étudiés.

[Comparative rough evaluations of noise figure for the four kinds of devices considered.]

\begin{tabular}{|l|c|c|c|c|c|}
\hline & $\begin{array}{c}\text { Profil } \\
\text { uniforme }\end{array}$ & $\begin{array}{c}\text { Profil } \\
\text { implanté }\end{array}$ & $\begin{array}{c}\text { Profil à } \\
\text { canal enterré }\end{array}$ & $\begin{array}{c}\text { Profil à } \\
\text { canal enterré }\end{array}$ & \\
\hline$N F(\mathrm{~dB})$ & 1,25 & 1,15 & 1,18 & 1,05 & $F=20 \mathrm{GHz}$ \\
$N F(\mathrm{~dB})$ & 2,9 & 2,48 & 2,40 & 2,20 & $F=40 \mathrm{GHz}$ \\
$N F(\mathrm{~dB})$ & 4,7 & 3,95 & 3,75 & 3,5 & $F=60 \mathrm{GHz}$ \\
\hline
\end{tabular}

$Z=75 \mu \mathrm{m}, L_{\mathrm{g}}=0.3 \mu \mathrm{m}, N_{\mathrm{d}}=2 \times 10^{17} \mathrm{at} / \mathrm{cm}^{3}, R_{\mathrm{s}}+R_{\mathrm{g}}=4,6 \Omega, I_{\mathrm{d}}=0,15 I_{\mathrm{dss}}$. 


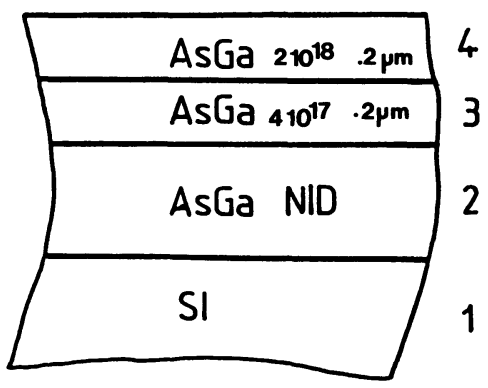

(อ)

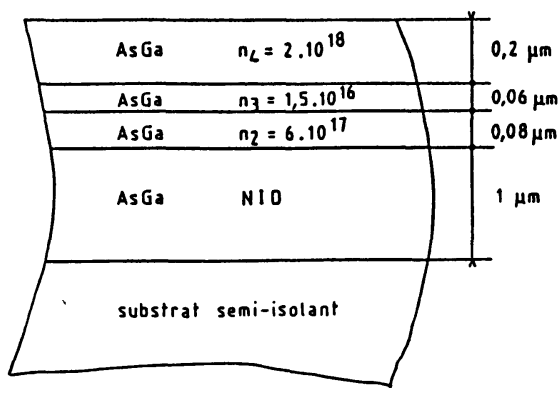

(b)

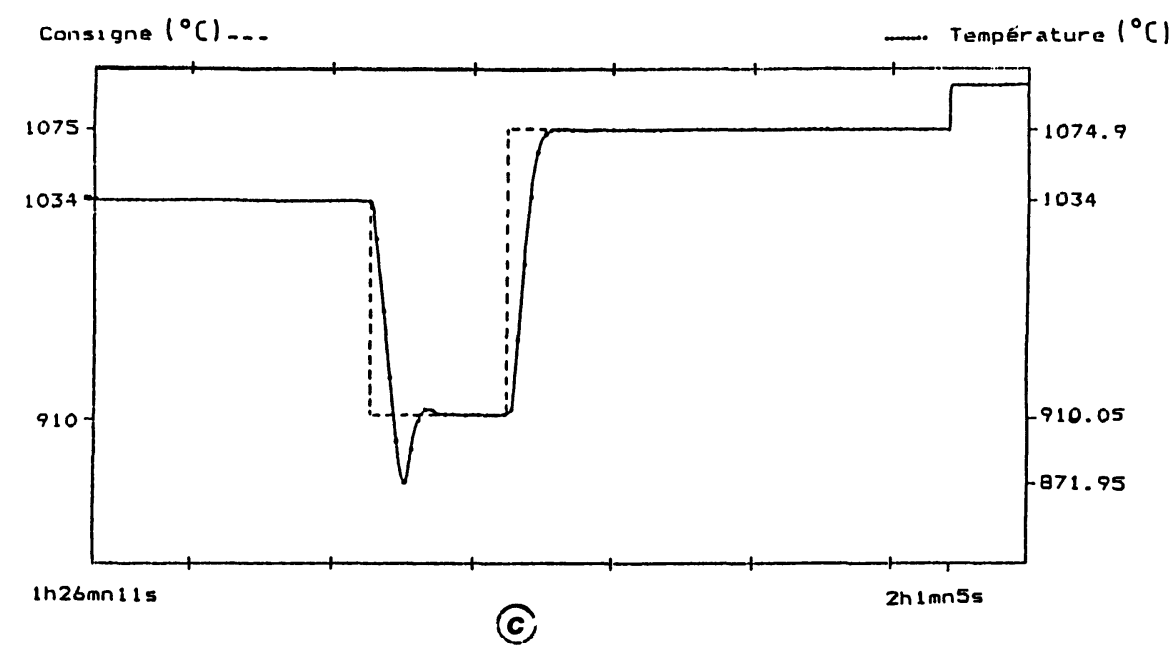

Fig. 16. - Couches élaborées par épitaxie par jets moléculaires : a) profil uniforme ; b) profil à canal enterré ; c) profil obtenu réellement pour la structure à canal enterré.

[Characteristics of the epilayers elaborated by M.B.E. : a) doping profile aimed ; b) buried channel ; c) real doping profile for the buried channel device.]

Enfin, on fait croître une couche très dopée de $2 \times 10^{18} \mathrm{at} / \mathrm{cm}^{3}$ sur une épaisseur de $2000 \AA$. Cette couche est destinée à obtenir une bonne qualité des contacts de source et de drain et à diminuer les résistances d'accès. La figure 16 représente les caractéristiques des différentes couches et la forme du profil réellement obtenu au niveau de l'épitaxie par jets moléculaires en ce qui concerne la structure à canal enterré.

Les principales étapes du processus technologiques ont été décrites largement par ailleurs [31]. Leurs caractéristiques essentielles sont : $0,2 \mu$

- structure recess de profondeur typique :

- longueur de grille : $1,3 \mu \mathrm{m}$;

- distance source drain : $4 \mu \mathrm{m}$;

— développements de grille de 75, 150 et $300 \mu \mathrm{m}$.

5.2 RÉSultats TYPiQues ObTENUS. - Nous avons représenté les caractéristiques essentielles comparatives des deux types de composants réalisés :

- caractéristique statique $I_{\mathrm{d}}=f\left(V_{\mathrm{gs}}\right)$ pour diverses valeurs de $V_{\text {ds }}$ (Fig. 17) ;
- les évolutions de la transconductance, de la conductance de sortie et de la capacité de réaction (Figs. 18 et 19) mesurées directement en microondes [39] en fonction de $V_{\mathrm{gs}}$;

- l'évolution correspondante de la fréquence de coupure $f_{\mathrm{c}}$ du gain en courant (Fig. 20). Signalons que si les valeurs des résistances d'accès $\left(R_{\mathrm{s}}\right.$ et $R_{\mathrm{d}}$ ) sont tout à fait convenables pour les composants à profil uniforme $(3 \Omega$ pour $Z=150 \mu \mathrm{m})$, elles sont nettement prohibitives pour les transistors à canal enterré $(7,5 \Omega$ pour $Z=150 \mu \mathrm{m})$. Ceci est lié au faible dopage de la couche de surface et au fait que nous ne disposions pas d'implantation ionique pour réaliser des zones surdopées.

L'analyse de ces résultats typiques nous permet de tirer les conclusions tout à fait conformes aux prévisions théoriques :

- les composants à canal enterré présentent une linéarité de comportement très supérieure à celle obtenue pour les transistors réalisés avec un profil uniforme : ceci apparaît clairement sur les évolutions de $g_{\mathrm{m}}$ et de $f_{\mathrm{c}}$ et montre bien tout l'intérêt de ces composants pour l'utilisation en puissance. Ceci 


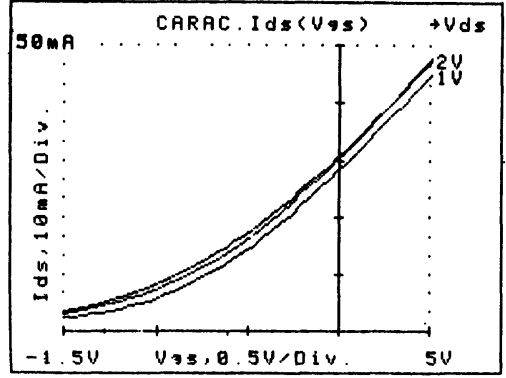

(a)

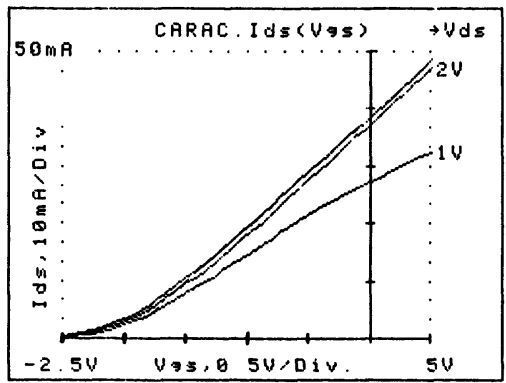

(b)

Fig. 17. - Caractéristiques expérimentales : a) profil uniforme ; b) profil à canal enterré.

[Comparison between the experimental characteristics $I_{\mathrm{d}}=f\left(V_{\mathrm{gs}}\right) ;$ a) uniform doping profile ; b) buried channel device.]

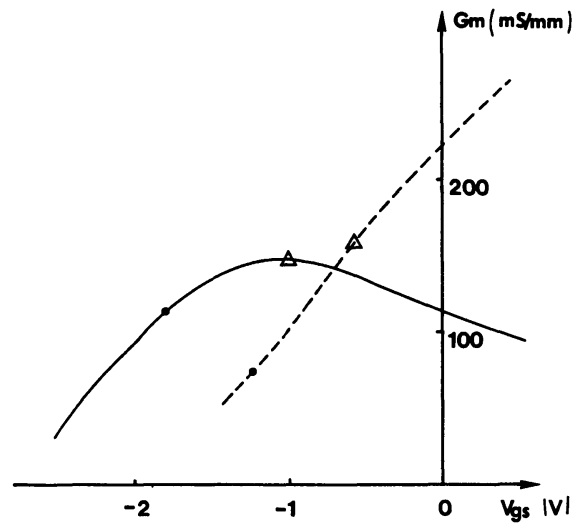

Fig. 18. - Evolution expérimentale de la transconductance $g_{\mathrm{m}}$ en fonction de la tension $V_{\mathrm{gs}}\left(V_{\mathrm{ds}}=3 \mathrm{~V}\right)$; - - profil uniforme ; - profil à canal enterré ; - conditions faible bruit; $\Delta$ conditions moyenne puissance.

[Comparison between the experimental transconductance variations $\left(V_{\mathrm{ds}}=3 \mathrm{~V}\right)$; - - - uniform doping device ; - buried channel device ; • low noise conditions; $\Delta$ large signal conditions.]
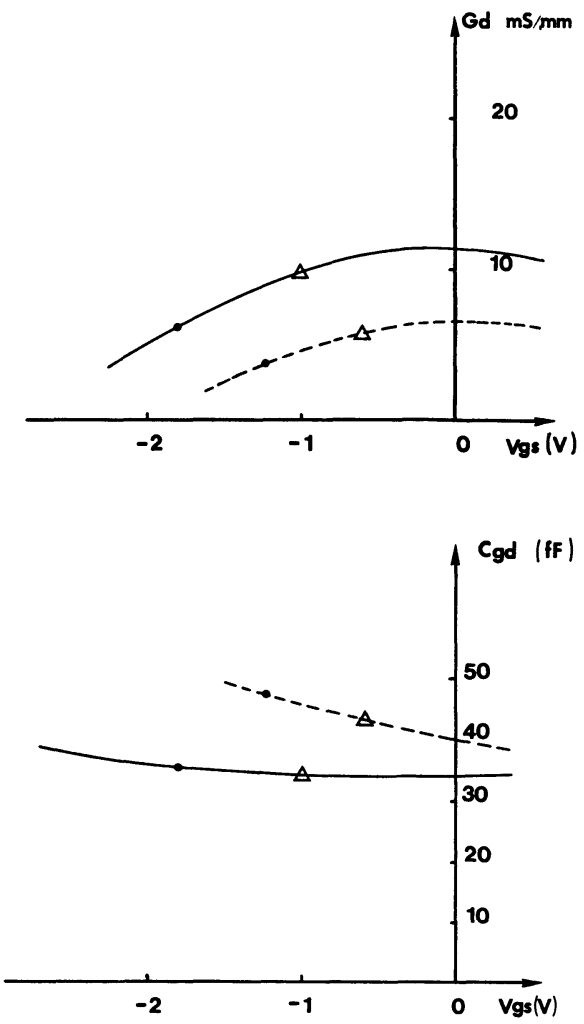

Fig. 19. - Evolution de la conductance de sortie et de la capacité de réaction $C_{\mathrm{gd}}$ en fonction de la tension $V_{\mathrm{gs}}\left(V_{\mathrm{ds}}=3 \mathrm{~V}\right) ;--$ - profil uniforme ; - profil à canal enterré ; $\bullet$ conditions faible bruit ; $\Delta$ conditions moyenne puissance.

[Experimental variations versus $V_{\mathrm{gs}}$ of the output conductance $g_{\mathrm{d}}$ and feedback capacitance $C_{\mathrm{gd}}$ in the same conditions as in figure 18.]

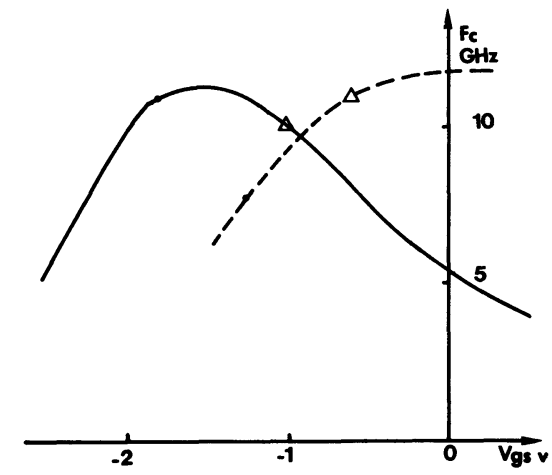

Fig. 20. - Evolution de la fréquence de coupure $f_{\mathrm{c}}$ en fonction de la tension $V_{\mathrm{gs}}\left(V_{\mathrm{ds}}=3 \mathrm{~V}\right)$. - - - profil uniforme; - profil à canal enterré ; • conditions faible bruit ; $\Delta$ conditions moyenne puissance.

[Comparisons between experimental cut-off frequency variations as function of $V_{\mathrm{gs}}$ in the same conditions as in figure 18.] 
confirme bien les résultats déjà publiés dans la littérature ;

- la supériorité potentielle des transistors à canal enterré en fonctionnement faible bruit est encore plus éclatante : l'augmentation de la fréquence de coupure est de $40 \%$, du rapport $f_{\mathrm{c}} / \sqrt{\boldsymbol{g}_{\mathrm{d}}}$ de l'ordre de $10 \%$ et la diminution du rapport $C_{\mathrm{gd}} / g_{\mathrm{d}}$ d'un facteur largement supérieur à 2. Malgré les valeurs élevées des résistances d'accès, l'amélioration du gain maximum utilisable est de l'ordre de $2 \mathrm{~dB}$ à $10 \mathrm{GHz}$. Nous n'avons pu tirer parti de cette supériorité potentielle au niveau du facteur de bruit à cause des valeurs trop élevées des résistances d'accès, qui déterminent directement les valeurs de ce paramètre, mais l'utilisation d'implantation localisée devrait sans difficulté notable permettre de résoudre ce problème.

\section{Conclusion.}

Nous avons développé un modèle de simulation numérique de transistor à effet de champ fonctionnant tant en régime statique que dynamique basé sur une résolution bidimensionnelle des équations des semiconducteurs, incluant les effets de dynamique non stationnaire. En particulier, le caractère opéra- tionnel d'un tel modèle a été clairement démontré. Il est possible d'en déduire à la fois les caractéristiques statiques et les paramètres dynamiques en régime petit signal en particulier en utilisant la réponse à un échelon de tension et la transformée de Fourier rapide. Ce modèle nous a permis de mettre en évidence l'important intérêt potentiel de composants à canal enterré tant pour l'amplification de puissance que pour l'utilisation en régime faible bruit. Ces prévisions théoriques ont été largement confirmées par les premiers résultats de l'étude expérimentale.

\section{Remerciements.}

Une partie importante de ce travail rentrait dans le cadre d'un contrat CNET-DAII effectué en collaboration avec la société AERO, le LEP et la DAG de Thomson-CSF. Nous tenons à remercier tout particulièrement Messieurs Mottet, Viallet et Caquot du CNET et Messieurs Berthon et Bouyer de la société AERO pour les nombreuses discussions concernant ce modèle, qui ont contribué à son perfectionnement. Nous remercions également les membres des Centrales de Technologie et de Caractérisation du Centre Hyperfréquences et Semiconducteurs sans qui la partie expérimentale de ce travail n'autait pu être réalisée.

\section{Bibliographie}

[1] Ruch, J. G. et KINO, G. S., Phys. Rev. 174 (1968) 921.

[2] Constant, E., Int. Phys. Conf. 57 (1981) 141.

[3] Statz, H., Haus, H. A. et Pucel, R. A., ieEe Trans. Electron. Devices 21 (1974) 549.

[4] ReISER, M., IEEE Trans. Electron. Devices 21 (1974) 549.

[5] Yamaguchi, K. et Kodera, H., IEEE Trans. Electron. Devices 23 (1976) 545.

[6] Frey, J. et WADA, T., Electron. Lett. 15 (1979) 28.

[7] WARriner, R. A., Solid State Electron. Devices 1 (1977) 105.

[8] Moglestue, C., Solid State Electron. Devices 3 (1979) 133.

[9] Zimmermann, J. et Constant, E., Solid State Electron. 23 (1980) 915.

[10] Bonjour, P. et al., IEEE Trans. Electron. Devices 27 (1980) 1019.

[11] Fauduembergue, R. et al., Physica 129B (1985) 563.

[12] Carnez, B. et al., J. Appl. Phys. 51 (1980) 784.

[13] El-Ghazaly, S. et al., Proc. 13th ESSDERC Canterbury (1983) p. 127.

[14] El-SAyed, O. L. et al., Can. J. Phys. 63 (1985) 727.

[15] SAlmer, G. et al., 11th Int. Symp. GaAs and Related compounds, Biarritz (1984).

[16] El-SAYED, O. L., et al., Solid State Electron. 30 (1987) 643.

REVUE DE PHYSIQUE APPLIQUÉE. - T. 23, N 7, JUILLET 1988
[17] Curtice, W. R. et Yuan, Y. H., IEEE Trans. Electron. Devices 28 (1981) 954.

[18] COOK, R. K. et Frey, J., IEEE Trans. Electron. Devices 29 (1982) 970.

[19] Brewitt-Taylor, C. R. et al., Boole Press Dublin (1979) p. 415.

[20] Snowden, C. M. et LoRET, D., IEEE Trans. Electron. Devices 34 (1987) 212.

[21] Mottet, S. et al., Nase Code IV Conference Dublin (1985).

[22] Archambaud, Y. et al., Nase Code VI Conference Dublin (1987).

[23] CAPPy, A., Thèse de $3^{\mathrm{e}}$ cycle, Lille (1981).

[24] Ibrahim, M. M., M. Sc. Thesis Cairo University (1983).

[25] Nougier, J. P. et al., J. Appl. Phys. 52 (1981) 825.

[26] Minondo, P., Thèse de $3^{\mathrm{e}}$ Cycle, Toulouse (1983).

[27] Le Mouellic, C., Thèse de $3^{\text {e }}$ Cycle, Rennes (1984).

[28] Mottet, S., Viallet, J. E., Nase Code IV Conference Dublin (1985).

[29] Viallet, J. E., Thèse de Doctorat, Paris VI (1985).

[30] Makram-Ebeid, S., Mimondo, P., Acta Electron. 25 (1983) 241.

[31] Heliodore, F., Thèse de Doctorat, Lille (1986).

[32] Heliodore, F. et al., 14th Int. Symp. on GaAs and Related Compounds (1987). 
[33] Shawkı, T. A. et al., 14th Int. Symp. on GaAs and Related Compounds (1987).

[34] LAUX, S. E., IEEE Trans. Electron. Devices 32 (1983) 2028.

[35] FukUTA, M. et al., IEEE Trans. Electron. Devices 23 (1976) 388.
[36] SAlmer, G. et al., Rapport de contrat DAII (1985).

[37] CAPPY, A., Thèse d'Etat Lille (1986).

[38] Dekkers, J. J. M. et al., IEEE Trans. Electron. Devices 28 (1981) 1065.

[39] Dambrine, G. et al., Ann. Télécommun. (à paraître). 